Convergences francophones $2.2(2015): 57-67$

http://mrujs.mtroyal.ca/index.php/cf/index

\title{
La pratique du surtitrage en contexte minoritaire
}

\author{
Milane Pridmore-Franz \\ University of Alberta
}

Le surtitrage est un genre de Traduction Audiovisuelle (TA) qui, depuis son essor sur la scène de la Compagnie d'opéra canadienne à Toronto en 1983, est rapidement devenu la forme dominante de la traduction théâtrale (Griesel 2005: 64) sur les scènes internationales. Les surtitres sont employés pour permettre les transferts culturels et langagiers sur diverses scènes dans le monde en préservant la pièce de théâtre dans sa langue et sa forme originales. En raison de leur efficacité et de leur rentabilité, les surtitres sont une méthode employée couramment par des compagnies théâtrales pour élargir leur public, surtout dans des contextes internationaux, tels que les festivals de théâtre ou les théâtres situés dans des destinations touristiques, ainsi que dans certains contextes minoritaires, tels que dans les théâtres francophones de l'Ouest canadien.

Au Canada, la pratique du surtitrage joue un rôle qui mérite une attention particulière. Un phénomène fascinant se déroule sur les scènes de l'Ouest canadien depuis au moins les huit dernières années. De Vancouver à Toronto, quatre théâtres professionnels francophones qui se trouvent en contexte minoritaire intègrent des surtitres anglais à leurs représentations de façon régulière afin d'élargir leur public et de satisfaire les besoins de familles exogames. Avec le Théâtre français de Toronto, qui se situe au centre du Canada, les théâtres de l'Ouest canadien dont $L a$ Troupe du jour à Saskatoon, L'UniThéâtre à Edmonton et Théâtre la seizième à Vancouver, se situent dans des villes de l'Ouest canadien où l'anglais est la langue dominante et où les communautés francophones sont moins présentes que dans les régions de l'Est. On peut ajouter à cette liste Le Cercle Molière à Winnipeg, où on intègre des surtitres pour certaines pièces de théâtre depuis au moins quelques années, mais pas de façon régulière.

Comme pour toute forme de traduction, on crée des surtitres en fonction du public visé. Ceci dit, la question du public cible se complique davantage lorsqu'il s'agit de surtitres puisque, comme Yvonne Griesel l'a mis en évidence, le public se compose de membres qui parlent ou comprennent la langue source (LS), de membres qui parlent ou comprennent la langue cible (LC) et de ceux qui parlent ou comprennent les deux langues (LS + LC) (2005: 67). Dans le contexte canadien, c'est une question et une condition primordiale: les surtitres ne sont pas conçus uniquement pour un public cible anglophone, mais aussi pour un public bilingue. Outre cet aspect, le contexte minoritaire de la langue et de la culture francocanadienne complexifie davantage le processus de surtitrage.

Compte tenu des enjeux sociolinguistiques et politico-culturels qui existent dans ces situations minoritaires, il se pourrait que les besoins des publics canadiens méritent une attention encore plus importante que dans des contextes de surtitrage conventionnels. Comme Henrik Gottlieb l'explique, le sous-titrage interlingual a sûrement plusieurs implications sociétales, politiques et linguistiques (2004: 87). Ce commentaire s'applique également à la pratique de surtitrage interlingual et s'avère encore plus important pour le contexte théâtral, puisque le théâtre est un médium culturel par excellence. Pour ces raisons, je dirais que l'emploi des surtitres 
dans de tels contextes minoritaires exige une plus grande sensibilité ainsi qu'une plus grande prise de conscience des circonstances sociolinguistiques, socioculturelles et sociopolitiques de la part de la traductrice.

Jorge Días Cintas affirme qu'on peut utiliser la traduction audiovisuelle comme moyen de promouvoir le multilinguisme et le multiculturalisme dans les pays ayant des communautés de plus en plus diverses et hétérogènes, mais aussi comme outil pour favoriser l'intégration sociale (6). Puisque les pièces de théâtre sont normalement enracinées dans un contexte culturel spécifique, les surtitres jouent un rôle intermédiaire entre deux langues et cultures, et possèdent également une capacité intrinsèque de promouvoir la compréhension et l'appréciation interculturelle et interlinguistique. Voilà pourquoi le surtitrage théâtral dans le contexte canadien hors Québec est un sujet d'importance. Les surtitres permettent aux spectateurs de s'immerger dans une représentation théâtrale en langue française, en écoutant la LS et en lisant simultanément les surtitres dans la LC (l'anglais). Nathalie Ramière déclare que les spectateurs de produits audiovisuels en traduction se retrouvent dans une position interculturelle (62), mais cet aspect s'avère encore plus pertinent dans le cas des surtitres pour le théâtre puisque le public prend physiquement part à une expérience culturelle réelle. Dans les contextes francophones minoritaires du Canada, les spectateurs regardent de véritables personnes sur la scène, entendent des accents régionaux et sont exposés à des pièces écrites par des dramaturges locaux ou régionaux. Les surtitres permettent donc une certaine ouverture et une plus grande accessibilité et visibilité des cultures francophones et plus concrètement, de la culture franco-canadienne dans des communautés qui se retrouvent en contexte anglo-dominant. Les surtitres peuvent ainsi servir d'outil pour promouvoir à la fois une meilleure connaissance et appréciation de la culture franco-canadienne, ainsi que l'apprentissage de la langue française au sein d'une culture principalement anglophone.

Compte tenu de la situation complexe et atypique qui se manifeste sur les scènes des communautés francophones minoritaires canadiennes, évoquée dans les paragraphes précédentes, comment aborde-t-on le processus de création des surtitres afin de mieux accommoder les différents publics cibles et assurer la meilleure réception du spectacle? Ceci, tout en prenant soin de ne pas encombrer ou déranger l'expérience des francophones et en maximisant également le transfert culturel et linguistique de la représentation aux spectateurs anglophones.

Cet article présente donc une analyse réflexive de mon approche du surtitrage, en tenant en compte des enjeux sociolinguistiques et politico-culturels qui influent sur les conditions de réception dans de telles situations linguistiques minoritaires. Cette analyse figure dans le cadre plus large d'une étude sur la réception des surtitres que j'ai effectuée en collaboration avec L'UniThéâtre entre octobre 2014 et avril 2015'. L'objectif de cette étude est de développer des stratégies de surtitrage qui amélioreront l'expérience des spectateurs et qui répondront mieux aux besoins des publics bilingues et non-francophones. Cette étude vise également à explorer le potentiel qu'offrent les surtitres pour l'apprentissage d'une deuxième langue (le français ou l'anglais). Les données

\footnotetext{
${ }^{1}$ Ce projet a été financé par le Conseil de recherches en sciences humaines (CRSH) du Canada.
} 
collectées par le biais de cette étude me permettront d'élaborer un cadre pour décrire la réception des surtitres dans des contextes multilingues, de fournir des précisions sur les aspects techniques liés aux surtitres et à raffiner le modèle de spectateurs développé par Griesel (2005: 67). Mon hypothèse est que ce modèle est encore plus nuancé dans des contextes bilingues, où les membres du public ont de différents niveaux de bilinguisme. Pour cette raison, les divers spectateurs utiliseront et percevront les surtitres de manières différentes.

C'est surtout sur cette dernière question que je me pencherai au cours de cette analyse réflexive. Sous l'angle de la dimension socioculturelle du contexte minoritaire et bilingue du public cible, je m'interrogerai sur mon rôle en tant que surtitreuse à $L$ 'UniThéâtre en abordant mes procédés de surtitrage et en examinant l'efficacité de ma stratégie. Je terminerai en ouvrant des pistes de réflexion à ce sujet et en évaluant de façon préliminaire si cette stratégie est bien adaptée en vue d'une meilleure réception de la part du public dans ce contexte bilingue minoritaire.

\section{Dimension socioculturelle : publics cibles et aspects culturels et linguistiques}

Il est bien connu qu'il existe un clivage historique entre les cultures francophones et anglophones au Canada. Tandis que 56,9 \% de la population canadienne dit avoir l'anglais comme langue maternelle, la proportion de la population parlant français est considérablement inférieure, soit 21,3\% (la grande majorité de cette population résidant au Québec). Notons que le nombre de bilingues ayant comme langue maternelle les deux langues officielles ne constitue qu'un maigre $0,4 \%$ de la population (Statistique Canada 2011). ${ }^{2}$ Pourtant, il faudrait tenir compte du fait que la majorité des francophones habitant dans des provinces ou territoires autre que le Québec sont pour la plupart bilingue par nécessité, donc si l'on envisage que $13,5 \% 3$ des locuteurs au Canada qui ont indiqué avoir appris le français comme langue maternelle habitent hors Québec, on peut estimer qu'il y aurait, selon les données du recensement de 2011, au moins 14 $\%$ de Canadiens bilingues (Statistique Canada 2011). Ainsi, nous devons considérer le fait que certains citoyens qui ont indiqué que leur langue maternelle est le français ne parlaient pas majoritairementle français à la maison. ${ }^{4}$

\footnotetext{
${ }^{2}$ Il faut préciser que les pourcentages ont été calculés à partir des données qui figurent sous la section «Langue maternelle » du Tableau thématique des données pour la population Canadienne. Il faut aussi noter que si l'on prend en compte le total des citoyens parlant l'anglais et une langue non-officielle (396 330), le pourcentage de locuteurs parlant l'anglais comme langue maternelle remonte à $58,1 \%$, et également, si l'on intègre le total de personnes parlant le français et une langue non-officielle (74 430) comme langue maternelle, le pourcentage de locuteurs parlant le français remonte à $21,5 \%$. Pareillement, si l'on prend en compte le nombre de personnes parlant comme langue maternelle l'anglais, le français, ainsi qu'une autre langue non-officielle (24 095), le pourcentage de bilingues (anglais/français) remonte à $0,5 \%$.

${ }^{3}$ Ce pourcentage a été calculé en soustrayant le nombre de personnes habitant au Québec qui ont appris le français comme langue maternelle (6 102 210) du nombre total de Canadiens qui ont indiqué avoir appris le français en tant que langue maternelle (7 054 970).

4 Tandis que 7054970 Canadiens ont indiqué avoir le français comme langue maternelle, 6827 865 ont indiqué que le français est la langue parlée le plus souvent à la maison.
} 
En raison de la grande disparité qui existe entre la présence du français et de l'anglais au Canada, plusieurs Franco-canadiens à l'extérieur du Québec cherchent à préserver leur identité culturelle et linguistique et à se protéger contre l'assimilation (linguistique et culturelle). C'est dans l'Ouest du Canada que la langue et culture franco-canadienne se retrouvent le plus exposées à la domination de l'anglais. Louise Ladouceur résume très bien cette situation : " Living in small communities, [francophones] have learned to coexist with the vast English majority while continuing to speak, write and create in French. They easily switch from one language to the other, but continue to claim French as their language of identity [and] the language with which they want to be identified, as bilingual Francophones » (Ladouceur 2009: 134).

En dépit de cette description, j'aimerais aussi suggérer que ce sentiment d'identité n'est pas identique pour tous les francophones. Plusieurs francophones bilingues qui sont nées dans les provinces de l'Ouest grandissent dans un contexte bilingue, ayant au moins un parent francophone. Les francophones bilingues qui demeurent dans l'Ouest canadien peuvent donc ainsi avoir un double sens identitaire, ou un « métissage identitaire » comme Rodrigue Landry, Réal Allard et Kenneth Deveau (2013) le définissent, avec différents degrés d'attachement à l'une ou l'autre des langues et cultures. Dans son examen sociologique du sens identitaire des communautés francophones canadiennes hors Québec, Joseph Yvon Thériault propose qu'il existe une "indécision identitaire» ou bien une "fragmentation identitaire » de la part de ces communautés minoritaires (17-18).

Malgré cette possibilité, l'explication de Ladouceur synthétise le phénomène global dans l'Ouest canadien, où le théâtre, étant une forme d'expression artistique culturellement enracinée, joue un rôle spécial au sein de ces petites communautés francophones (Ladouceur 2009: 132), car le théâtre est un milieu privilégié d'affirmation identitaire et de résistance culturelle face à la domination de l'anglais dans l'espace public (2013: 113-4). Tandis que le théâtre est un médium important où résonnent la langue et la culture franco-canadiennes (112), la réalité est que les théâtres francophones ont du mal à subsister dans un environnement qui se compose d'un public minoritaire. Ajoutons à cela le grand nombre de théâtres anglophones qui existent à Vancouver, Edmonton, Saskatoon, Winnipeg et Toronto, et la situation se complique davantage. Voilà la raison pour laquelle l'emploi des surtitres dans les théâtres francophones de l'Ouest canadien (ou du Canada Central dans le cas de Toronto) qui souhaitent rejoindre un vaste public anglophone auparavant inaccessible, est une stratégie qui a connu un succès remarquable (119).

Les théâtres francophones tels que L'UniThéâtre à Edmonton, où je suis chargée de la création des surtitres sous la supervision de la Professeure Louise Ladouceur depuis 2012, non seulement élargissent leur auditoires à des publics non-francophones ou bilingues en intégrant des surtitres à leurs productions, mais ils permettent également à leurs spectateurs francophones d'apprécier une représentation dans leur langue maternelle en compagnie de leur conjoint ou de membres de leur famille qui ne parle(nt) pas nécessairement le français. Pareillement, les surtitres offrent aux francophiles ou aux apprenants du français, la possibilité de faire l'expérience de la langue et de la culture franco-canadienne 
ou française dans une situation passive, où ils peuvent s'engager avec la langue dans un contexte réel, sans devoir la parler eux-mêmes. Les surtitres offrent donc une véritable occasion culturelle à plusieurs genres de spectateurs et rendent accessibles le théâtre francophone à un public divers.

La population francophone d'Edmonton est assez limitée : 68,1 \% des Edmontoniens qui ont participé au recensement de Statistique Canada de 2011 ont déclaré l'anglais comme étant leur langue maternelle, alors que $2 \%$ ont affirmé que leur langue maternelle est le français et un maigre $0,3 \%$ ont indiqué qu'ils sont bilingues, parlant les deux langues officielles comme langue maternelle. ${ }^{5}$ Notons que ces francophones parlent fort probablement l'anglais en tant que langue seconde et sont pour la plus part parfaitement bilingues. Pour souligner l'étendue réelle de la situation francophone minoritaire à Edmonton, nous n'avons qu'à remarquer que selon le recensement de 2011, 98,9\% plus d'Edmontoniens parlaient l'anglais à la maison que le français (Statistique Canada 2012). ${ }^{6}$

Compte tenu de l'importance du rôle du théâtre francophone, on peut imaginer que dans une ville dominée par l'anglais, il se pourrait que certains francophones aient une certaine résistance à la présence de surtitres anglais. (En fait, métaphoriquement parlant les surtitres dominent la scène, puisqu'ils sont le plus souvent affichés sur un écran situé au-dessus de la scène.) Mes hypothèses sont que les surtitres peuvent affecter l'expérience du public francophone de multiples façons : 1) soit les surtitres sont perçus comme étant redondants ou inutiles et/ou représentent une source de distraction ; 2) soit certains membres ont une aversion totale envers les surtitres et ne sont pas d'accord avec le fait qu'ils soient employés dans un théâtre francophone ; 3) soit que certains membres francophones aient parfois recours aux surtitres pour faciliter leur compréhension. Un fait dont je ne doute pas, c'est que le public bilingue francophone jugera les choix de traduction et la qualité des surtitres, puisqu'ils sont dans la position de pouvoir comparer les dialogues et le message livré par les surtitres. Ladouceur affirme qu'on doit assurer que les surtitres soient conformes au texte source pour ne pas détourner l'attention des membres du public qui peuvent comprendre les messages livrés dans les deux langues, car toute disparité serait une source de distraction (2013: 119).

Il est important de garder à l'esprit que le type de stratégie employé influencera la perception de la culture cible à l'égard de la culture source (Ramière

\footnotetext{
${ }^{5} \mathrm{Il}$ faut préciser que les pourcentages ont été calculés à partir des données qui figurent dans la section «Langue maternelle détaillée » du tableau du Profil de recensement pour la ville d'Edmonton. Il faut aussi noter que si l'on prend en compte le total pour les citadins parlant l'anglais et une langue non-officielle (16 525), le pourcentage de locuteurs parlant l'anglais comme langue maternelle remonte à $70,1 \%$, et également, si l'on intègre le total de personnes parlant le français et une langue non-officielle (945), le pourcentage de locuteurs parlant le français comme langue maternelle remonte à $2,1 \%$. Si l'on prend en compte le nombre de personnes parlant l'anglais, le français, ainsi qu'une autre langue non-officielle, le pourcentage de bilingues (anglais/français) demeure à $3 \%$.

${ }^{6}$ « Langue parlée le plus souvent à la maison », données pour la ville d'Edmonton : 634740 personnes ont indiqué que l'anglais était la langue qu'ils parlent le plus souvent à la maison, tandis que seulement 6550 personnes ont indiqués qu'elles parlant le français en tant que langue parlée le plus souvent à la maison.
} 
156), ainsi que la perception de la part des spectateurs à l'égard de la pièce de théâtre. Puisque les surtitres sont un produit multisémiotique (Mateo135-6) qui n'est jamais complet dans l'absence des autres aspects théâtraux (dialogue, éclairage, trame sonore, projections, costumes, etc.) nous pouvons seulement juger la traduction en tenant compte des autres fonctions de la représentation. Comme je le montrerais par la suite, celles-ci peuvent également influencer les surtitres, parfois au détriment de leur qualité.

Quant à la traduction des surtitres, ce qu'on jugerait comme étant des modifications sémantiques ou sémiotiques acceptables dans la culture cible pourrait trahir le texte source (Gottlieb 2005: 15). Les incohérences entre le texte source (le dialogue et la mise en scène) et le texte cible surtitré pourrait être plus néfaste que bénéfique, surtout dans des contextes culturellement et linguistiquement minoritaires.

\section{La création des surtitres: enjeux, perceptions, reception}

Griesel décrit le sutritrage comme étant une forme créative et littéraire, mais parallèlement, une forme de traduction pragmatique, puisque les surtitres servent une fonction communicative et artistique (2009: 122). La surtitreuse doit s'assurer que les surtitres s'accorderont avec le genre particulier et les qualités intrinsèques de la représentation, en prenant soin de ne pas détruire l'œuvre d'art dans la version traduite (122). Elle devrait ainsi prendre en considération son public cible avant de déterminer ses stratégies de traduction. Une surtitreuse doit toujours envisager la diversification du public et ne peut ignorer aucun groupe de spectateurs (122). Si la surtitreuse s'occupe aussi de la projection des surtitres, elle doit ainsi se concentrer attentivement sur la scène et assurer une bonne synchronisation, tout en demeurant imperceptible.

Voilà un défi des surtitres. Même si ceux-ci s'accordent minutieusement au texte source sur la scène, la précision des surtitres va dépendre de celle des comédiens livrant leur dialogue. Les surtitres étant un produit fixe qu'on ne peut pas modifier en direct, au moins dans le cas de théâtres comme L'UniThéâtre qui utilisent PowerPoint comme mode de diffusion, on peut difficilement s'adapter à des modifications spontanées dans le dialogue. Même si les surtitres sont soigneusement segmentés et que la surtitreuse assure une bonne synchronisation avec le dialogue parlé sur scène, s'il y a un moment imprévu et qu'un acteur saute une ou plusieurs lignes, renverse l'ordre du dialogue ou décide d'improviser, la synchronisation et le contenu des surtitres ne s'accorderont plus à la représentation. Dans de telles circonstances, les spectateurs ne savent pas que les comédiens se sont trompés et pensent automatiquement que les surtitres sont erronés, voire de mauvaise qualité.

Si l'on considère tous ces aspects, associés aux complexités culturelles des productions théâtrales et des différents groupes sociolinguistiques des spectateurs, la pratique du surtitrage constitue un processus de traduction hybride véritablement complexe. Griesel explique très bien ce fait: « [Surtitling is] a very complex interlingual transfer between different cultures, different theatre cultures, between interpretation and translation, between intercultural and international theatre, between oral and written words, between a divided audience, and intermedial and 
multimedial theatre » (2009: 122). Comme pour la traduction conventionnelle, les surtitreurs doivent avoir une très bonne connaissance des cultures source et cible, et posséder des compétences linguistiques et interculturelles, car ils doivent bien interpréter les significations socioculturelles dans des rencontres transculturelles. De cette façon, les surtitreurs contribuent au développement culturel et au transfert de connaissances d'une culture vers une autre (Muñoz-Calvo 2-3). Linda Dewolf attache une grande importance à la fonction culturelle des surtitres et encourage les traducteurs à expérimenter avec les potentiels interculturels que ceux-ci offrent, en soulignant qu'ils sont un moyen de préserver l'identité culturelle tout en créant un dialogue entre les diverses communautés culturelles et linguistiques (s.p.). Pour sa part, Yves insiste ainsi sur le fait que les surtitreurs jouent et joueront un rôle majeur s'ils réalisent pleinement leur fonction socioculturelle (2006: 7). Dans le contexte minoritaire de l'Ouest canadien, la surtitreuse devrait avoir connaissance des potentiels interculturels qu'offrent les surtitres afin d'exploiter ces possibilités et créer des connexions entre les cultures francophones et anglophones canadiennes en utilisant des stratégies de traduction qui préserveront le texte et la culture source.

En tant que surtitreuse, je me perçois comme étant une médiatrice interculturelle. $\mathrm{Au}$ vu du contexte socioculturel et sociolinguistique canadien, mon hypothèse est que la meilleure manière d'aborder la création des surtitres dans de telles circonstances est de préserver le contenu et la forme du texte source en utilisant une approche plus ou moins littérale, selon les limites imposées par les contraintes traductives et techniques associées au surtitrage ${ }^{7}$ et à la traduction en général. Mon objet principal en utilisant une telle stratégie est de préserver la primauté du texte source et d'exploiter la nature culturelle et linguistique de la représentation.

Je perçois le contenu et la forme comme étant deux éléments importants pour la cohérence interne d'une pièce de théâtre, donc je privilégie les complexités du dialogue et de la représentation. De cette manière, les surtitres n'ont pas une fonction purement communicative. Normalement, la création de surtitres exige une réduction du texte source de façon à ne pas trop empiéter sur les autres aspects sémiotiques représentés sur la scène. Ceci dit, les surtitres ne sont pas une simple suggestion - les spectateurs dépendent des surtitres pour bien saisir les nuances du spectacle (Griesel 2009: 121). On peut considérer ma stratégie de surtitrage comme étant une forme hybride entre la stratégie de condensation (qui implique aussi les stratégies de 'compensation', 'paraphrase', 'adaptation', 'reformulation' et 'omission') et de la stratégie conventionnelle de traduction littérale.

On pourrait appeler une telle stratégie la «traduction condensée-directe », puisqu'il est toujours nécessaire de réduire le texte cible dans une certaine mesure à cause des contraintes spatio-temporelles des surtitres. Généralement, le degré de condensation qu'on apporte au texte cible dépendra du montant de dialogue livré sur scène, ainsi que du rythme du dialogue (Griesel 2005: 71). Cependant, si l'on réduit le texte ou omet complètement des segments du texte source, cela pourrait conduire à une perte d'informations et à des déformations stylistiques. C'est la raison pour laquelle Griesel affirme que la surtitreuse devrait évaluer les

\footnotetext{
${ }^{7}$ Voir Orero et Matamala « Accessible Opera: Overcoming Linguistic and Sensorial Barriers » pp. 268 pour étudier un tableau sur les défis du surtitrage.
} 
conséquences de son choix et de ses méthodes de traduction (71) et réfléchir à la manière dont les surtitres affecteront les publics cibles.

Dans le contexte minoritaire canadien, omettre des informations dans les surtitres n'est pas un choix idéal, puisque plusieurs membres, sinon la majorité, sont bilingues et remarqueront qu'il y a un décalage entre le dialogue et les surtitres. Dans les contextes de surtitrage internationaux, on observe peut-être moins cette règle. Il est préférable de conserver les valeurs culturelles du texte source dans la mesure du possible, dans des limites raisonnables pour ne pas faire obstacle à la compréhension du public non-francophone. La surtitreuse peut réaliser cet objectif en utilisant un genre de traduction sourcière en vertu de laquelle les qualités culturelles comme les noms propres ou les jurons ayant une connotation culturelle demeurent intacts dans la traduction.

Cette méthode de « traduction condensée-directe » est une stratégie utile qui sert à équilibrer les deux couches linguistiques de la représentation. Les similarités qui existent entre les traits linguistiques du français et de l'anglais facilitent l'application d'une stratégie littérale, mais évidemment, les références culturelles imposent souvent une situation de non-équivalence en traduction. Dans de tels cas, la surtitreuse va devoir adapter le texte source d'une façon à reproduire le sens et l'aspect connotatif du dialogue de manière qui sera compréhensible pour le public non-francophone.

\section{Réflexions sur la stratégie de traduction choisie}

On vient de voir qu'une telle stratégie peut préserver les caractéristiques linguistiques et culturelles d'une pièce de théâtre. En revanche, cette stratégie risque d'être contradictoire et contre-intuitive à l'objectif conventionnel du surtitrage, qui est de réduire la charge cognitive de la part des spectateurs et d'éviter que les surtitres soient une source de distraction. Il est possible d'arriver à un tel résultat en réduisant le texte cible surtitré. La fonction centrale des surtitres est de permettre aux spectateurs de suivre l'intrigue et de comprendre les émotions des personnages sur la scène tout en demeurant relativement discrets (Orero et Matamala 267). Estella Oncins souligne que la majorité des professionnels et des chercheurs conviennent que les surtitres doivent être brefs, simples et composés de structures claires (12). Néanmoins, l'utilisation d'une stratégie plus littérale entraîne automatiquement des segments de textes plus longs et parfois plus verbeux. Les spectateurs doivent par conséquent lire plus de texte et ont moins de temps pour se concentrer sur la représentation. En utilisant une telle stratégie, la surtitreuse crée des segments de surtitres de deux à trois lignes de jusqu'à 40-45 caractères (avec espaces) par ligne. Oncins affirme que la forme la plus courante est de deux lignes de texte par segment et un maximum d'environ 35 caractères par ligne, mais elle mentionne aussi que certains théâtres et opéras ont des surtitres de trois lignes au maximum, avec 40 caractères par ligne (12).

Le contexte minoritaire dans l'Ouest canadien présente une dichotomie pour la surtitreuse, qui doit choisir entre deux possibilités: 1) elle peut préserver les éléments culturels, linguistiques et artistiques du texte source et exploiter les potentiels culturels des surtitres. Cette option sert à privilégier le message de la représentation, mais éventuellement au détriment des autres aspects sémiotiques du 
texte source à cause de la longueur des surtitres; 2) la deuxième option est de privilégier la représentation dans sa globalité avant la fonction communicative en réduisant le texte cible pour permettre aux spectateurs de se concentrer autant que possible sur la scène.

Il sera intéressant, et aussi bénéfique aux recherches actuelles sur le surtitrage, de déterminer si la stratégie de «traduction condensée-directe » est une stratégie appropriée pour des représentations théâtrales et pour les publics spécifiques à ce contexte minoritaire. Les résultats de cette première étude sur la réception des surtitres devraient apporter la lumière sur les aspects et contraintes techniques du surtitrage. Par le biais d'un sondage, les spectateurs de L'UniThéâtre ont, au cours de la saison théâtrale 2014-2015, été consulté en ce qui concerne notamment leur perception de la qualité et quantité de texte des surtitres, leur synchronisation, leur temps de lecture, leur lisibilité, et le degré d'attention qu'ils suscitent sur la scène.

Je présume qu'en dépit de la possibilité que les surtitres conçus pour les représentations à L'UniThéâtre puissent exiger une plus grande attention de la part des spectateurs en raison de leur longueur, ils empêchent minimalement le public de se concentrer sur la scène. Les stratégies de surtitrage choisies à L'UniThéâtre devraient remplir leur fonction en tant qu'outil de promotion de la langue et de la culture franco-canadiennes en fournissant des potentiels pédagogiques et en préservant la nature de la représentation. On peut prévoir la publication de mon mémoire résumant les résultats de l'étude sur la réception des surtitres à L'UniThéâtre en Décembre de cette année. Les résultats de cette étude apporteront un éclairage nouveau sur la manière dont les publics réagissent aux surtitres et nous aideront par conséquent à mieux adapter les surtitres pour des publics bilingues et multilingues. 


\section{Bibliographie}

Dewolf, Linda. « La place du surtitrage comme mode de traduction et vecteur d'échange culturel pour les arts de la scène. » Theatre Research in Canada / Recherches théâtrales au Canada 24.1 (2003): s. p. Web. 18 déc. 2014

Díaz-Cintas, Jorge. " Audiovisual Translation Comes of Age. » Between Text and Image: Updating Research in Screen Translation. dir. Delia Chiaro, Christine Heiss \& Chiara Bucaria. Amsterdam: John Benjamins, 2008. 112.

Gambier, Yves. « La traduction audiovisuelle: un genre en expansion. » Meta: Journal des traducteurs/Meta: Translators' Journal 49.1 (2004): 1-11. Web. 13 juin 2013.

---. « Multimodality and Audiovisual Translation. » MuTra 2006 - Audiovisual Translation Scenarios: Conference Proceedings (2006): 1-8. Web. 14 juin 2013.

---. « Sous-titrage et apprentissage des langues. » Linguistica Antverpiensia, New Series-Themes in Translation Studies 6 (2007): 97-113. Web. 13 juin 2013.

---. « Challenges in Research on Audiovisual Translation. » Translation Research Projects 2 (2009): 17-9. Web. 13 juin 2013.

Gottlieb, Henrik. «Language-Political Implications of Subtitling. » Topics in Audiovisual Translation. Éd. Pilar Orero. Amsterdam: John Benjamins, 2004. 83-100.

---. « Multidimensional Translation: Semantics Turned Semiotics. » Challenges of Multidimensional Translation (2005): 33-61. Web. 14 juin 2013.

Griesel, Yvonne. " Surtitles and Translation. Towards an Integrative View of Theatre Translation. » EU High Level Scientific Conference Series (2005): 62-75. Web. 14 juin 2013.

---. « Surtitling: Surtitles another Hybrid on a Hybrid Stage. » TRANS: revista de traductologia 13 (2009): 119-27. Web. 14 juin 2013.

Ladouceur, Louise. « Bilingualism on Stage: Translating Francophone Drama Repertoires in Canada. » TRANS: revista de traductología 13 (2009): 12936. Web. 14 juin 2013.

---. « Exploring a Bilingual Aesthetics through Translation in Performance. » Translation in Theatre and Performance (2013): 111-29.

Landry, Rodrigue, Réal Allard et Kenneth Deveau. « Bilinguisme et métissage identitaire: vers un modèle conceptuel. » Minorités linguistiques et société /Linguistic Minorities and Society 3 (2013): 56-79.

Mateo, Marta. " Surtitling Today: New Uses, Attitudes and Developments. » Linguistica Antverpiensia 6 (2007): 35-154. Web. 14 June 2013.

Muñoz-Calvo, Micaela. "Introduction. » Translation and Cultural Identity: Selected Essays on Translation and Cross-Cultural Communication. dir. Micaela Muñoz-Calvo and Carmen Buesa-Gómez. UK: Cambridge Scholars Publishing, 2010. 1-12. Web. 15 déc. 2014.

Oncins, Estella. «The Tyranny of the Tool: Surtitling Live Performances. » Perspectives: Studies in Translatology (2013): 1-20. Web. 20 avril 2014.

Orero, Pilar et Anna Matamala. «Accessible Opera: Overcoming Linguistic and 
Sensorial Barriers. » Perspectives: Studies in Translatology 15.4 (2007): 262-77. Web. 14 juin 2014.

Ramière, Nathalie. « Reaching a Foreign Audience: Cultural Transfers in Audiovisual Translation. » The Journal of Specialized Translation 6 (2006): 152-66. Web. 17 déc. 2014.

Statistique Canada. «Recensement de la population de 2011, produit numéro 98314- XCB2011058 au catalogue de Statistique Canada. » Gouvernement du Canada, 2011. Web. 5 août 2015.

---. « Edmonton, Alberta (Code 0252) et Alberta (Code 48) (tableau). Profil du recensement, Recensement de 2011, produit $\mathrm{n}^{\circ}$ 98-316-XWF au catalogue de Statistique Canada. » Gouvernement du Canada, 2012. Web. 5 août 2015.

Thériault, Joseph Yvon. « Entre la nation et l'ethnie: sociologie, société et communautés minoritaires francophones. » Sociologie et sociétés 26.1 (1994): 15-32. Web. 14 juin 2014. 